\title{
New technologies for feminine hygiene products with reduced environmental impact
}

\author{
G. Carlucci \\ $P \& G$, Italy
}

\begin{abstract}
Since their first introduction at the beginning of the 20th century, disposable feminine hygiene products have been developed just focusing on improving their functional performance. Up until recently, only a few attempts were made to create eco-friendly alternatives, but their higher costs and lower performance have greatly limited the relative market potential.

Our research focused on creating more sustainable products greatly reducing the use of materials and the creation of waste but still delivering all functional benefits required by the consumers (absorbency, dryness, flexibility, comfort, etc.). of:

The newly designed disposal products offer environmental benefits in terms

o increasing the use of renewable resources thanks to the introduction of highly performing bio-based superabsorbent polymers, which will reduce the use of oil-based absorbent materials;

o reducing the volume and mass of the absorbent materials with a consequent benefit in terms of less waste disposal and a reduction in the required packaging material;

o decreasing emissions (less trucks circulating per year) related to the transporting of materials, products and disposal phases.

We wish to present how product optimization can be combined with biobased materials paying-off in terms of sustainability, performance and competitiveness.

Keywords: disposable products, feminine hygiene, absorbency, sustainable design, bio-based material.
\end{abstract}




\section{Introduction}

Disposable feminine hygiene products had their genesis at the beginning of the $20^{\text {th }}$ century, when wood pulp bandages were used to create pads with easily obtainable and cheap materials. Successful commercialization commenced in the 1920 s and since then the use of disposable feminine hygiene products has become pervasive in the industrialized world. These products were mainly based on cellulose as the absorbent component. This is linked to the capability of cellulose to be easily wetted by water and aqueous liquids thanks to its abundance in hydroxyl hydrophilic groups. In late 1970s and early 1980s disposable feminine hygiene products development was influenced by the introduction of new materials such as cross-linked polyelectrolytes, e.g. polyacrylate super absorbent polymers. In fact these polymers have much improved fluid retention in comparison with cellulose. Currently, these products are manufactured and commercialized in all countries of the globe. In 2010 the global volume of sales was about 220 Billion of units and it is forecast to grow by about $18 \%$ in 2015 .

These products typically comprise a liquid-pervious topsheet as wearer-facing layer, a backsheet as garment-facing layer and an absorbent core structure, between topsheet and backsheet. The body fluids are acquired through the topsheet and subsequently stored in the absorbent core structure. The backsheet typically prevents the absorbed fluids from wetting the wearer's garment.

An absorbent core structure typically comprises fibrous absorbent materials, such as natural, man-made and synthetic fibers. Further, it comprises superabsorbent polymers (SAP), in particulate form dispersed among the fibers, in order to improve the fluid retention characteristics. SAP typically are waterinsoluble, water-swellable, hydrogel-forming crosslinked absorbent polymers (e.g. polyacrilates) [1].

Along the years the development of these products has been focused on improving their functional performance for protection and comfort. Only few efforts were made to create eco-friendly alternatives.

For example, there were attempts to use natural-based materials such as agar, alginates, pectin, gums, carboxyalkyl cellulose, chitin [2] but they have not gained usage for market expansion in such articles because their absorbent properties are inferior compared to SAP and because their costs are much higher than current materials.

Another big limitation for eco-friendly designs is linked to the process of the industrial production of disposable feminine hygiene products. This process mainly consists of the combination and integration of the above functional materials that usually comes in rolls from suppliers. In order to have a profitable and reliable production, the single layer manufacturer produces the materials in continuo on machines several meters wide. The output material is then slit to the desired width before shipment. This choice forces the materials to be homogeneous across the width and/or the length of the product. This limitation is true across the different technologies of material used. Raw material homogeneity is strongly limiting the design flexibility and consequently the 
optimization opportunities delivering advantages for overall sustainability balance.

\section{Technology development}

Many efforts have had to be made to create environmental improvements, in order to guarantee concurrent high level technological standards and market satisfaction. Hereby we present the main achievements reached.

Our R\&D program demonstrated that actions for mitigating the environmental impact in the absorbent products for feminine hygiene sector can be developed through an optimized combination of bio-based materials, ecodesign technologies, and new engineering process.

Our R\&D activities led to new technologies with the potential to substantially reduce use of materials and waste improving the environmental performance.

One of those technologies concerns a high performing and bio-based super absorbent material specifically designed for the absorption of menses to replace and/or reduce SAP materials due to its much higher menses absorption capabilities combined with appropriate cost scenario. In light of reducing oilbased materials we developed a series of bio-based polymers demonstrating their ability to block menses. The most promising ones for an industrial application (cost/performance ratio, safety, technology readiness) belong to the cationic starches category. Our cationic starches interact with menses components creating a gel which is then strongly entrapped into the structure. The absorption is many times better than a standard SAP. The application on this highly effective bio-based material positioned in the key area of the core structure makes it possible to partially substitute SAP components with positive consequence in terms of the amount of renewable resources used and environmental performance. Furthermore, this application enables an estimated reduction of 5\% in total material usage and of 5\% in packaging material.

Another fundamental piece of the new sustainable product is a multilayer core structure with highly optimized component design that fosters component synergies. Thanks to high design flexibility, this new core structure also enables the localization of the components (e.g. SAP and/or cationic starches) in all the axis of the composite.

The new multilayer core structure brought to reality the opportunity of breaking the previously mentioned boundaries of current technologies, designing multilayer structure where the absorbent materials can be positioned in the core according to desired designs and patterns. This allows main advantages:

- by having each individual layer forming the new structure exploiting the various functions of a core, it is possible to push the material optimization;

- by leveraging the designed multilayer absorbent core to position the absorbency exactly and only where it is needed and in the right quantity according to possible different solutions.

The combination of the bio-based material with the design flexibility provided by the engineered multilayer structure the innovative aspects powered by our development. 
We are currently up-scaling the new technologies for the material and the manufacturing process to a pre-industrial scale. We are targeting 20-30\% material usage reduction, a saving of $10 \% \mathrm{t}_{\mathrm{CO} 2 \mathrm{eq}} / \mathrm{yr}$ per year and an overall significant waste prevention.

\subsection{Bio-derived super absorbent material}

In the past we discovered [3] that the combination of chitosan material together with an SAP in disposable feminine hygiene products delivers improved absorption performance as this combination was particularly effective in the case of electrolyte-containing solutions like menses.

Firstly, the menses retention characteristics of chitosan materials are due to the presence in the polymer structure of ionizable cationic functional groups. These groups are usually ammonium groups, a high proportion of which are in salt form when the polymer is dry but which undergo dissociation upon contact with bodily fluid. In the dissociated state, the polymer chain will have a series of functional groups attached to it which groups have the same electric charge (e.g., $-\mathrm{NH}_{3}{ }^{++} \mathrm{H}_{3} \mathrm{~N}-$ ) and thus repel one another. This leads to expansion of the polymer structure, which, in turn permits further absorption of negatively charged molecules present in menses.

Secondly, the positively charged cationic groups of the chitosan materials will interact with negatively charged anionic groups-bearing molecules present in bodily fluids, like the carboxylic groups of proteins or hydroxylic acid bearing entities like short chain acid (e.g., butyric acid). This will result in the formation of tri-dimensional net between chitosan materials and such molecules with anionic groups (gelification of the bodily fluids).

Preferred chitosan materials used are chitosan salts as, due to a higher percentage of positively charged cationic groups, they deliver all above mentioned benefits immediately upon contact with bodily fluids.

However, the high cost of chitosan materials has prevented until now their commercial uses. In fact, despite chitosan and its chemistry being known about for centuries [4, 5], its source (mainly from shrimps and crabs) is still cumbersome and limited.

From the above considerations we work to create a material having high ability to immobilize menses at an affordable price. We developed specific cationic modified starches with unique combination of characteristics that deliver performance comparable to chitosan derivatives in disposable feminine hygiene products. The modified starches are synthesized by using low level of crosslinking agent and are water soluble. These cationic starches perform particularly well in the presence of proteinaceous fluids such as menses and deliver fluidhandling benefits, and have better ability to increase the viscosity of blood-based fluids than other starch derivatives already described. Cationic modified starches have the further advantage to come from a raw material (starch) largely available and cheaper compared to chitosan salts.

Our cationic modified starches are the product of the reaction between starch and a suitable cationizing agent comprising an ammonium group, wherein the degree of substitution of the cationizing agent is less than 0.50 . The cationic 
modified starch is cross-linked by a cross-linking agent, wherein the crosslinking agent is reacted in concentration less than $4000 \mathrm{ppm}$ by weight of starch. The cationic modified starches have a net positive charge in aqueous solutions at a $\mathrm{pH}$ range from 5 to 9 .

The source of starch before cationic modification can be chosen from many sources including corn starch, wheat starch, rice starch, waxy corn starch, oat starch, cassaya starch, waxy barley, tapioca starch, potato starch. Starch comprises two distinct polymer types made of glucose units. One type of polymer is amylose whereas the other is amylopectin. Our new cationic starch was made with a starch comprising amylopectin at a level above $95 \% \mathrm{Wt}$.

The cationic agents used in the fabrication of the modified starches comprise an ammonium group and are:

○ 2,3-epoxypropyl-N,N,N-trimethylammonium chloride (available as a $70 \%$ aqueous solution under the name QUAB 151),

- 3-chloro-2-hydroxypropyl-N,N,N-trimethylammonium chloride (available as a $65 \%$ aqueous solution under the name of QUAB 188),

○ 3-chloro-2-hydroxypropyl-N,N,N-dimethylethanolammonium chloride (available as a 65\% aqueous solution under the name of QUAB 218)

The extent of the cationization of the polysaccharide by the cationizing agent is expressed by using the degree of substitution of the reactive groups of the polysaccharide by the cationizing agent (referred as the "degree of substitution of the cationizing agent"). The cationic modified starches have a degree of substitution of cationizing agent in the range of from about 0.15 to about 0.40 .

The modified starches are relatively soluble and provide the desired properties. We mean that they contain less than $75 \%$ of insoluble carbohydrate. The solubility data can be measured as follows: the modified starch $(1 \mathrm{~g})$ is slurried in distilled water $(100 \mathrm{ml})$ at room temperature $\left(21^{\circ} \mathrm{C}\right)$ with stirring for 15 minutes. The slurry is allowed to stand for 8 hours before filtering. The dissolved carbohydrate in the filtrate is measured by the known colorimetric method employing the use of the phenol/sulphuric acid test for soluble carbohydrate. In these determinations to $1 \mathrm{ml}$ of the sample of the test solution is added $1 \mathrm{ml}$ of phenol solution $(5 \% \mathrm{w} / \mathrm{v})$ followed by $5 \mathrm{ml}$ of concentrated sulphuric acid and the liquids mixed by hand shaking for one minute. After leaving to cool for an hour the concentration of the soluble carbohydrate is determined using a ultra-violet spectrophotometer from the absorbance at the peak at $483 \mathrm{~nm}$ by reference to a glucose standard.

The concentration of cross-linking agent of from about $200 \mathrm{ppm}$ to $3000 \mathrm{ppm}$ in the reaction mixture is advantageous to obtain the desired amount of crosslinking. By "ppm" we mean the relative amount of the cross-linking agent expressed in weight units per weight of the starch material to be cross-linked expressed in parts per million. Suitable cross-linking agents are epichlorohydrine and phosphorusoxychloride.

Quaternary modified starches were synthesized according to Heinze et al. [6]. We used as solvent ethanol instead of isopropanol. The way to compare the effectiveness of the modified starches in immobilizing menses was done by rheological analysis as indicated below. 
Samples preparation: a 5\% w/w water solution of the cationic modified starch is prepared weighting $0.25 \mathrm{~g}$ of cationic starch powder into a Petri dish of $12 \times 12$ $\mathrm{cm}$. Then $4.75 \mathrm{~g}$ of distilled water are added and the mixture is stirred until a homogeneous gel is obtained into the Petri dish. After gelling, the Petri dish is placed into a ventilated oven with temperature set at $45^{\circ} \mathrm{C}$. for $8 \mathrm{hrs}$ until complete dryness. After drying into the Petri dish, a layer of dried cationic modified starch powder will be present. The Petri dish is removed from the oven and kept 1 hour to equilibrate at room temperature. To the dried cationic polymer layer are slowly added $4.75 \mathrm{~g}$ of menses simulating fluid wetting the entire surface. After the addition, the material is further mixed with a spatula for $5 \mathrm{~min}$ until a homogeneous gel is obtained.

Evaluation of rheological properties: Rheology parameters $\left(G^{\prime}\right)$ has been measured using parallel plate geometry of $40 \mathrm{~mm}$ with a gap of $2 \mathrm{~mm}$ at $40 \mathrm{~Hz}$, at $37^{\circ} \mathrm{C}$. The instrument was a stress tech HR supplied from Reologica Instruments Inc 231 Crosswicks Road Bordentown, NJ 08505 USA. This instrument was operated using a parallel plate geometry of $40 \mathrm{~mm}$ with a gap of $2 \mathrm{~mm}$. $\mathrm{G}^{\prime}$ is the elastic modulus and the values measured at $40 \mathrm{~Hz}$ are considered representative of the ability of the cationic modified starch to thicken artificial menses fluid. The higher the value $G^{\prime}$ the better the modified starch immobilizes menses in an absorbent product.

Menses simulating fluid is based on defibrinated sheep's blood that has been modulated to ensure it closely resembles human menstrual fluid in viscosity, electrical conductivity, surface tension and appearance. The relative characteristics lie in a range close to that of normal menstrual blood [7]. The viscosity should lie in the range of 7 to 8 (units cStK). The $\mathrm{pH}$ should lie in the range of 6.9 to 7.5 and the conductivity in the range 10.5 to 13 (units mmho).

\subsection{New absorbent core structure and design}

The new absorbent core comprises 2 substrate layers entrapping a discontinuous layer of absorbent polymer material particulates The discontinuous layer of absorbent material comprises a layer of thermoplastic material in partial contact with the surfaces of the substrate layers.

The layer of absorbent polymer material is distributed over the substrate layer with uniform or non uniform basis weight over the area interested by the distribution. The layer of absorbent polymer material can also be a discontinuous layer comprising openings, i.e. areas substantially free of absorbent polymer material, which are completely surrounded by areas comprising absorbent polymer material. These openings have a diameter of less than $10 \mathrm{~mm}$.

The layer of thermoplastic material is made of hot melt adhesive that serves to immobilize the absorbent polymer material. The adhesive is a fiberized hot melt adhesive, i.e., being provided in fibers as a fibrous layer. The hot melt adhesive is partially in contact with the absorbent polymer material and partially in contact with the substrate layer

In this structure the absorbent polymer material layer is provided as a discontinuous layer, a layer of adhesive is laid down onto the layer of absorbent polymer material such that the adhesive layer is in direct contact with the layer of 
absorbent polymer material but also in direct contact with the substrate layer where the substrate layer is not covered by the absorbent polymer material in correspondence of the openings of the discontinuous layer of the absorbent polymer material. This imparts an essentially three-dimensional structure to the fibrous layer of hot melt adhesive which in itself is a two-dimensional structure of relatively small thickness (in z-direction), as compared to the extension in $\mathrm{x}$ and $y$-direction. In other words, the layer of adhesive undulates between the absorbent polymer material and the substrate layer. The areas where the layer of adhesive is in direct contact with the substrate layer are the areas of junction. The areas of junction can be of squared, rectangular or circular shape. Areas of junction of circular shape can have a diameter of less than $10 \mathrm{~mm}$. The areas of junction can be disposed in a regular or irregular pattern. For example, the areas of junction may be disposed along lines. These lines should have a certain angle in respect to the longitudinal edges of the core. A disposition along lines parallel with the longitudinal edges of the absorbent core might create channels in the longitudinal direction which can lead to a lesser wet immobilization, hence the areas of junction should be arranged along lines which form an angle of about 20-45 degrees with the longitudinal edges of the absorbent core.

Thereby, the adhesive provides spaces to hold the absorbent polymer material towards the substrate layer and can thereby immobilize this material. In a further aspect, the adhesive is bond to the substrate thus affixing the absorbent polymer material to the substrate. Hot melt adhesive materials can also penetrate into both the absorbent polymer material and the substrate layer, thus providing for further immobilization and affixation.

Therefore the hot melt adhesive materials provides a very good immobilization of absorbent polymer material when the article is dry and it also delivers a much improved immobilization of absorbent polymer material when the article is wet or partially loaded.

The hot melt adhesive materials that are useful for immobilizing the absorbent polymer material, combine good cohesion and good adhesion behaviors. Good adhesion ensures that the hot melt adhesive layer maintains good contact with the absorbent polymer material and in particular with the substrate material. Good cohesion ensures that the adhesive does not break, in particular in response to external forces, and in response to strain. The adhesive is subject to external forces when the absorbent product has acquired liquid, which is then stored in the absorbent polymer material which in response swells. An exemplary adhesive allows for such swelling, without breaking and without imparting too many compressive forces, which would restrain the absorbent polymer material from swelling.

Preferred thermoplastic compositions meeting these requirements have the following features:

- the thermoplastic composition comprises a single thermoplastic polymer or a blend of thermoplastic polymers, having a softening point, as determined by the ASTM Method D-36-95 "Ring and Ball", in the range between $50^{\circ} \mathrm{C}$ and $300^{\circ} \mathrm{C}$; the thermoplastic polymer has typically a molecular weight $(\mathrm{Mw})$ of more than 10,000 and a glass transition temperature $\left(T_{g}\right)$ usually below room temperature. 
Typical concentrations of the polymer in a hot melt are in the range of $20-40 \%$ by weight. Such thermoplastic polymers are water insensitive.

Exemplary polymers are (styrenic) block copolymers including A-B-A triblock structures, A-B diblock structures and (A-B)n radial block copolymer structures wherein the A blocks are non-elastomeric polymer blocks and the $\mathrm{B}$ blocks are unsaturated conjugated diene or hydrogenated versions of such. The B block is typically isoprene, butadiene, ethylene/butylene (hydrogenated butadiene), ethylene/propylene (hydrogenated isoprene). The resin of the hotmelt adhesive has an $\mathrm{Mw}$ below 5,000 and a $\mathrm{T}_{\mathrm{g}}$ above room temperature, the concentrations of the resin are in the range of 30-60\%. The plasticizer has a low Mw of less than 1,000 and a $\mathrm{T}_{\mathrm{g}}$ below room temperature, the concentration is 0 $15 \%$. Low glass transition temperatures $T_{g}$ are beneficial for good adhesion. Furthermore, a low glass transition temperature $T_{g}$ ensures that the adhesive thermoplastic material does not become too brittle. The preferred adhesive has a high cross-over temperature $T_{x}$, that is beneficial for high temperature stability of the thermoplastic layer and hence it ensures good performance of the absorbent product and in particular good wet immobilization even under conditions of hot climates and high temperatures. Therefore, $\mathrm{T}_{\mathrm{x}}$ should preferably be above $90^{\circ} \mathrm{C}$.

\subsection{New absorbent core process}

Further it is fundamental that the process for creating the new core comprises the steps of:

- providing a substrate material,

- precisely depositing absorbent material onto the substrate material in a pattern that exactly reproduces the above described parameters,

- fiberizing a hot melt adhesive onto the substrate material and the absorbent material, such that portions of the hot melt adhesive are in direct contact with the substrate and portions of the hot melt adhesive are in direct contact with the absorbent material.

Specifically, the absorbent core is laid down onto a down drum, which presents an uneven surface. In a first process step, the substrate layer is laid on to the uneven surface. By using a vacuum means, the substrate layer material will follow the contours of the uneven surface and thereby the substrate layer material will assume a mountain and valley shape. Onto this substrate layer absorbent polymeric material is disposed. The absorbent polymer material will accumulate in the valleys presented by the substrate layer.

In a further process step a hot melt adhesive is placed onto the absorbent polymer material applied by a nozzle system. The nozzle system provides a relatively thin but wide curtain of adhesive. This curtain of adhesive is than placed onto the substrate layer and the absorbent polymer material. As the mountain tops of the substrate layer are less covered by absorbent polymer material the adhesive will make contact with these areas of the substrate layer.

In a further process step a cover layer is placed upon the substrate layer, the absorbent polymer material and the hot melt adhesive layer. The cover layer will be in adhesive contact with the substrate layer in the areas of junction. 
Hence, the uneven service of the lay-down drum determines the distribution of absorbent polymeric material throughout the storage layer and likewise determines the pattern of areas of junction.

The distribution of absorbent polymeric material is profiled and most preferably profiled in the longitudinal direction. Hence, along the longitudinal axis of the absorbent core, the basis weight of the absorbent polymer material will change. Preferably the basis weight of absorbent polymer material in at least one freely selected first square measuring $1 \mathrm{~cm} \mathrm{x} 1 \mathrm{~cm}$ is at least $30 \%$ higher than the basis weight of absorbent polymer material in at least one freely selected second square measuring $1 \mathrm{~cm} \times 1 \mathrm{~cm}$.

\section{Environmental benefits}

The new technologies maintain current absorbent performance, improving environmental impacts of the final product by a methodological approach based on three vectors:

- the application of the innovative bio-derived material will open the opportunity to use renewable materials and will reduce the overall material quantity for about $5 \%$ with reference to the current product values;

- the innovative multilayer absorbent core, which is based on a high degree of super absorbent material and an optimized design of the components will deliver $10-15 \%$ reduction of volume and mass;

- the optimization of SAP localization according to the requirements of the liquid distribution will give an incremental reduction of the required material up to an additional $10-15 \%$;

The specific environmental benefits from above introduction of bio-based material and material reduction are:

- the introduction of the innovative bio-derived (starch) material that will reduce the percentage of oil based particles in various areas of the multilayer structure. The objectives will be about $10 \%$ of percentage reduction of nonbiodegradable components;

- the potential to reduce the material and volume up to $25-30 \%$ per specific feminine hygiene products that will reduce the environmental problems of packaging (about $25 \%$ of packaging material reduction), transport (estimated in about $10 \% \mathrm{t}_{\mathrm{CO} 2 \mathrm{eq}} / \mathrm{yr}$ saving per year) and related waste disposal. The impact on the globe of such material and consequent waste reduction is even more relevant, considering the high quantity of consumption of these products. For instance in the West Europe 24 billions of disposal absorbents are currently produced every day. The results will provide benefit in the management of the landfills and waste disposal.

All the above improvements perfectly match with European policies and directives, specifically:

1. The need to reduce the environmental impacts of urban waste through prevention or reduction of its production according to Directive 2006/12/EC. In fact, the project will reduce the percentage of volume and mass of the absorbent core material up to $20-30 \%$ with a consequent benefit in terms of 
waste disposal, which is even more relevant considering the $20-25 \%$ reduction in the required packaging material.

2. The need for more sustainable production and consumption patterns aiming to ensure that consumption of renewable and non-renewable resources does not exceed the carrying capacity of the environment, as it is declared in the decision No 1600/2002/EC - Sixth Community Environment Action Program. In fact, the project increases the sustainability in the European industrial production of advanced absorbent materials for feminine hygiene products. With reference to the state of the art, relevant improvements are expected thanks to the reduction of product volume and mass. On top of saving in raw material (up to 20-30\%) there are, as consequences: a reduction of natural resources and emissions related to transport (up to 10\% reduction in the usage of the number of trucks per year), packaging (up to $20-25 \%$ with respect to the current packaging material usage) and disposal phases. Moreover, promising improvements are supposed in terms of renewable resourced usage (up to $20 \%$ ) thanks to the introduction of biobased particles, which will reduce the oil-based materials of the multilayer structure.

\section{Acknowledgement}

This project is pursued with the contribution of the financial instruments of EU LIFE+ Environment Policy \& Governance program 2010.

\section{References}

[1] Chatterjee, P.K. and Gupta, B. S., Absorbent Technology, Elsevier; Amsterdam, 2002.

[2] Dutkiewicz, J.K., Nonwoven structures for absorption of body fluids, Edana; Brussels, 2003.

[3] Gagliardini, A., et al., US Patent 6887564, 2005.

[4] Muzzarelli, R.A.A. and Peter, M.G., Chitin Handbook, European Chitin Society; 1997.

[5] Muzzarelli, R.A.A. and Muzzarelli, C., Chitosan in Pharmacy and Chemitry, Atec Edizioni; Italy, 2002.

[6] Heinze, T., Haack, V., Rensing, S., Starch Derivatives of High Degree of Functionalization. 7. Preparation of Cationic 2-Hydroxypropyltrimethylammonium Chloride Starches. Starch Stärke 56, pp. 288-296, 2004.

[7] Bussing, H. J., Zur Biochemie des Menstrualblutes. Zentrablatt fur Gynaekologie 79, pp. 456-560, 1957. 